\title{
Modelo de Enseñanza-Aprendizaje para el Estudio de la Cinemática de un Volante Inercial usando Tecnologías de la Información y la Comunicación en un Laboratorio de Física
}

\author{
Claudio M. Enrique y Gloria E. Alzugaray \\ Grupo de Investigación en Enseñanza de la Ingeniería (GIEDI); UDB Física, Dpto. Materias Básicas, \\ Dpto. Ingeniería Mecánica; Universidad Tecnológica Nacional, Facultad Regional Santa Fe, Lavaisse 610, \\ 3000 Santa Fe, Argentina (e-mail: cenriquear@yahoo.com.ar)
}

Recibido Jun. 05, 2012; Aceptado Jul. 23, 2012; Versión final recibida Sep. 23, 2012

\begin{abstract}
Resumen
El objetivo de este trabajo fue analizar la cinemática de un volante inercial en un laboratorio de un curso universitario de física usando Tecnologías de la Información y la Comunicación (TIC's). Se determinaron simultáneamente la posición, la velocidad y la aceleración angular de dicho volante en función del tiempo. Se empleó un equipamiento basado en las TIC's que consistió en un dispositivo conformado por el volante, una pesa, una cuerda inextensible y un equipo de adquisición de datos en tiempo real conformado por un sensor de rotación, una interfase, una computadora y un programa informático. Los resultados han permitido diseñar un Trabajo Práctico de Laboratorio de la asignatura Física I en el que se enseña a los estudiantes a tratar el tema como un trabajo de investigación. Por lo tanto se les solicita a los estudiantes que documenten el informe, argumenten la discusión y concluyan sobre los principales aspectos del estudio.
\end{abstract}

\section{Learning-Teaching Model for studying the Kinematics of an Inertial Steering Wheel using Information and Communication Technologies in a Laboratory of Physics}

\begin{abstract}
The goal of this work was to analyze the kinematics of a inertial steering wheel in a laboratory of a university course of physics using Information and Communication Technologies (ICT`s). The angular position, speed and acceleration of the steering wheel as a function of time were simultaneously determined. An equipment based on ICT's that consisted of a device that included the steering wheel, a weight, an inextensible rope and a real time data acquisition system that considered a rotation sensor, an interface, a personal computer and an computer program. The results have allowed designing a Practical Work of Laboratory for a first course of physics in which the students are taught to treat the subject as a research work. Thus, they have to review the literature, present a good discussion and conclude on the mains aspects of the study.
\end{abstract}




\section{INTRODUCCIÓN}

Las Tecnologías de la Información y la Comunicación (TIC's) pueden ser usadas como herramientas potencializadoras en la enseñanza de la Física experimental, aunque su uso debe hacerse de manera consciente y reflexiva. Es claro que la tecnología por sí sola no implica una buena educación. Pero sin duda, es casi imposible conseguir una buena educación sin tecnología (D'Ambrosio, 2003). El uso de las TIC's en los Trabajos Prácticos de Laboratorio (TPL's) de Física deben provenir de la redefinición y la reorientación del concepto de trabajo práctico, y de una mejor adaptación de la actividad al objetivo escogido. En ocasiones, es conveniente considerar que la enseñanza de la Física, o de las ciencias experimentales en general, constan de tres aspectos principales: i) El aprendizaje de la ciencia, adquiriendo y desarrollando conocimientos teóricos y conceptuales; ii) El aprendizaje sobre la naturaleza de la ciencia, desarrollando un entendimiento de la naturaleza y los métodos de la ciencia, siendo conscientes de las interacciones complejas entre ciencia y sociedad; y iii) La práctica de la ciencia, desarrollando conocimientos técnicos sobre la investigación científica y la resolución de problemas.

Para asegurar que los estudiantes tengan éxito en el aprendizaje sobre la naturaleza de la ciencia, el primer paso es convertir lo implícito en explicito. El segundo paso necesario es que exista una planificación conforme a un modelo científico desde una paradigma educativo caracterizado por un espacio constructivista del aprendizaje, un modelo de ciencia que valorice la falibilidad y la dependencia teórica de la observación y del experimento, que aporte conciencia de cómo se transmiten los conocimientos dentro de la comunidad científica, y que haga hincapié en la distinción entre teorías -cuyo objetivo es explicar- y modelos instrumentalistas, que persiguen realizar predicciones y establecer una medida de control (Hodson, 1993). En muchos casos los experimentos pueden simplificarse mediante la eliminación de algunos pasos menos importantes y el empleo de aparatos y técnicas más sencillas. Esto se puede ver facilitado mediante el uso de las TIC's, principalmente aquellas que trabajan con un sistema de adquisición de datos informatizados y que permita medir alguna magnitud física en tiempo real.

El docente de Física, o de ciencias en general, debe apropiarse de las TIC's para poder hacer una abstracción de sus enormes potencialidades con el objeto de realizar un análisis crítico orientado a lo que estos recursos permiten o inhiben en términos de aprendizajes, a fin de entender la acción educativa que debería incorporar elementos de reflexión docente tales como contenidos, estrategias, tareas, estructuras de conocimiento; los contextos social, institucional y aúlico, etc. Para ello es necesario la creación de nuevos escenarios donde, amén de la interpretación y comprensión de códigos y lenguajes de las áreas de conocimiento y de los recursos, sino también de la enseñanza que se integre a los procesos tradicionales, junto a la selección de información u otras acciones que se integren a los procesos activos de construcción de saberes. También es fundamental del dominio de aspectos pedagógicos específicos para poder adaptar las propuestas didácticas a su entorno particular de enseñanza y poder realizar nuevos diseños poniendo en juego la creatividad. Consideramos que "la transformación de las formas de enseñar no se produce por la renovación de los artefactos, sino por la reconstrucción de los encuadres pedagógicos de dicha renovación (Maggio, 2000).

Acordamos con Litwin (2003) en que la tecnología limita o enmarca, potencia o banaliza la propuesta pedagógica y didáctica. Por ello la capacitación debe orientarse al desarrollo de la capacidad de autogestión del docente para la selección, diseño e implementación de propuestas didácticas, realizadas sobre un análisis crítico tanto de objetivos educativos, recursos materiales y humanos, como del contexto aúlico, institucional y jurisdiccional. La cuestión no es "cuánto y cómo" se interactúa externamente con el recurso, sino cuánto promueve el uso del mismo los procesos que llevan a comprender, explicar, predecir, transformar el conocimiento; lo cual implica proveer desde el recurso, con estrategias didácticas apropiadas, a la modificación de las estructuras del conocimiento, de las representaciones internas del sujeto, con el objeto de promover la adquisición de significados científicos compartidos y significativos (Ausubel et al, 1989).

\section{LAS TIC'S EN LA ENSEÑANZA DE LA FÍSICA}

En el espacio concerniente a la enseñanza de la Física en general y en particular en las carreras de Ingeniería, aún no se ha logrado superar una primera fase donde se proponen experiencias de laboratorio o estrategias para utilizar las TIC's en clases teóricas o en clases de resolución de problemas y se investigue, salvo honrosas excepciones (Pontes, 2001) sobre su valor como estrategia educativa, aún cuando hay advertencias que se asientan en la intuición (Sanmartí e Izquierdo, 2001), sobre las ventajas y desventajas de utilizar las TIC's. Es conocido que la práctica docente responde a distintas finalidades, y que su diseño debe realizarse de acuerdo al objetivo planteado para la misma (Capuano, 2006a; Capuano 2006b). También se puede decir que una vez diseñada la práctica docente su arquitectura definirá los aprendizajes, y no escapa a esta consideración la presencia de las TIC's en distintos aspectos de la misma. 
Uno de las situaciones más comunes en los TPL's de Física consiste en analizar de manera individual variables que podrían interconectarse con otras para analizar más exhaustivamente al sistema desde un punto de vista integral. Por ejemplo, suele estudiarse a un movimiento partiendo de que si es con aceleración lineal constante, es uniformemente acelerado. Si bien es válido para situar el estudio desde una referencia, normalmente suele omitirse lo que sucede con la velocidad y/o la posición. Pero en los problemas, estas variables se tratan de vincular entre sí, lo que habitualmente desconcierta al alumno sobre todo cuando inicia sus estudios en Física y quiere resolver todo con "la fórmula".

\section{EL USO DE LAS TIC'S EN EL LABORATORIO DE FÍSICA}

En particular, en este trabajo se considera la elaboración de buenos materiales (Litwin, 2008) para la enseñanza que implica la posibilidad de reutilización de los mismos, su adaptabilidad y versatilidad, para enfrentar diferentes situaciones según los destinatarios. Por lo tanto, el diseño y desarrollo de los materiales didácticos incluyendo TIC's puede constituir un campo de estudio, al considerarlos instrumentos de integración de contenidos, así como también herramientas ineludibles para el proceso de enseñanza aprendizaje, generando situaciones susceptibles de ser sometidas al análisis, evaluación e investigación permanente.

Los sistemas de adquisición de datos por PC presentan viarias desventajas: son generados y/o provistos por empresas particulares, generalmente requieren de la asistencia a cursos especiales por parte de los docentes que deseen operarlos, y la mayoría de las veces son algo onerosos. No obstante ello, su uso se compensa por la gran importancia que tiene para el proceso de enseñanza-aprendizaje en la experimentación directa del alumno con el sistema en estudio. Dicha actividad, siempre que sea abordada con una metodología adecuada, resulta insuperable en lo que hace a motivación y a la internalización de los fenómenos, elementos indispensables para la conceptualización de las distintas leyes y la generación de los modelos mentales correspondientes.

Las experiencias asistidas por computadora responden a una tecnología hoy ampliamente disponible, cuyo aprovechamiento para la enseñanza es conveniente desde diferentes puntos de vista. Sin embargo, no se trata sólo de una cuestión tecnológica, sino que presupone un definido enfoque pedagógico, en la medida que esta manera de llevar a cabo los experimentos, puede llegar a complementar, o incluso, reemplazar, los métodos tradicionales de laboratorio.

Dentro de las características fundamentales de las TIC's frente a las llamadas técnicas tradicionales de experimentación y medición, se pueden citar: a) mayor exactitud y precisión en general; b) mayor velocidad y/o frecuencia de adquisición de datos; c) posibilidad de procesamiento de datos en línea, o en forma inmediata; d) obtención casi automática de gráficas y resultados numéricos; y e) posibilidad de alcanzar una mayor motivación de los alumnos.

La mayor parte de estos aspectos pueden representar ventajas desde el punto de vista didáctico, siempre y cuando se considere la manera de abordar las aplicaciones. Si bien en los métodos tradicionales de TPL existe una especie de "anclaje" en la realidad - tal como medir el tiempo de determinada experiencia con un cronómetro digital - y resulte menos precisa que el uso de una interfase conectada a una PC, en esta última se puede "caer" en experiencias que pueden tornarse abstractas para el alumno donde no pueda comprender lo que hace si no le son explicados los fundamentos del método. Por otro lado, la Motivación Intrínseca definida por Larkin y Chabay (1996) como la voluntad a involucrarse en la actividad por sí misma y no por influencia de factores externos, depende de tres condiciones, que pueden darse o no de manera simultánea: desafío, curiosidad, y control. El diseño de los dispositivos experimentales y la modalidad de trabajo con los alumnos deberían contemplar estos aspectos. En el uso de las TIC's en los TPL debe orientarse dentro de un marco teórico sostenido por un enfoque constructivista del aprendizaje. Creemos que para realizar este enfoque, deben considerarse las siguientes propuestas:

Anclaje en conocimientos previos: para permitirle al alumno la combinación de cierta práctica tradicional con la experiencia automatizada, de modo que adquiera una noción clara de las magnitudes medidas, y una capacitación básica de manipulación de instrumentos tradicionales.

Control del sistema que estudia: para planificar la actividad de modo que sea el propio alumno quien manipule el sistema de experimentación y la computadora, éste debe estar instruido - aunque tal vez en forma sintética -, sobre los fundamentos de la técnica de adquisición y transferencia de datos entre el sistema y la computadora, de manera que no le resulten extraños los elementos electrónicos de conexión; diferenciar datos analógicos de digitales; y que conozca distintos accesos de la computadora para las conexiones exteriores. 
Curiosidad por conocer aspectos fundamentales y habitualmente desconocidos: aprovechando que las mediciones de la computadora son más rápidas, exactas y precisas en experiencias que implican una mayor complejidad, se pueden llegar a conclusiones que muchas veces son prácticamente inalcanzables a través de técnicas manuales. Esto provoca desafíos para resolver situaciones problemáticas que trasciendan la simple realización de operaciones absolutamente programadas, buscando por el contrario que el alumno pueda tomar decisiones en el desarrollo de la práctica experimental, para lo cual se podrán proponer problemas, preguntas o incógnitas a develar a través de la experimentación

Desafío entre diferentes equipos de trabajos, y discusión de los resultados con argumentaciones y refutaciones: se busca promover el trabajo colaborativo dentro de cada equipo de alumnos, y la comparación de los resultados encontrados entre distintos grupos, realizando una especie de confrontación entre los distintas comisiones de TPL.

\section{LOS TRABAJOS PRÁCTICOS DE LABORATORIO}

Según Richoux (2003) "la estructura "clásica" de los trabajos prácticos se apoya sobre el hecho de poner a disposición de los estudiantes una ficha de actividades y aparatos adecuados para estudiar diferentes fenómenos, generalmente de forma cuantitativa (mediciones, tratamientos numéricos, modelización)". Hodson (1994) presenta más de un tipo de TPL: "investigaciones personales poco estructuradas" y "ejercicios prácticos de acuerdo con un conjunto de indicaciones explícitas". El TPL en el proceso de enseñanza-aprendizaje es generalmente considerado como una actividad de aprendizaje útil para una diversidad de funciones (Barberá y Valdés, 1994; Lynch, 1987, Hodson, 1994; Andrés, 2002). Sin embargo, en la realidad se ve un marcado énfasis en el aprendizaje de destrezas y técnicas de recolección y procesamiento de datos experimentales, con pocas o ninguna relación explícita con referentes teóricos o modelos. Además, se vislumbra una falta de claridad en cuanto a los objetivos de aprendizaje que se espera lograr con el TPL. Esta inconsistencia entre la finalidad asignada al laboratorio en términos de aprendizaje y lo que se realiza hace en la práctica tanto en el aula (Andrés, 2002) como en la investigación (Tobin et al., 1994), no ha permitido evaluar la efectividad del laboratorio en la enseñanza. En tal sentido, es primordial precisar el rol del TPL en el contexto de la enseñanza de las ciencias experimentales. Diversos autores (Hodson, 1994; Duit, 1995; Barberá y Valdés, 1996; Sére, 2002) consideran que el TPL en la enseñanza de las ciencias es importante para: integrar lo conceptual y lo fenomenológico; establecer una conexión dialéctica entre datos y teoría; y promover el desarrollo de una visión de la naturaleza de la ciencia más cercana al quehacer científico.

En ciencia es difícil pensar en una actividad experimental desligada de las ideas o componentes teóricos que representan el mundo físico. Sin embargo, la relación teoría - práctica puede ser vista desde diferentes posiciones epistemológicas (Sére, 2002; Andrés e Pesa, 2002), las cuales se reflejarán en la acción didáctica. Una de la formas que toma la acción didáctica, es definida como que la práctica ayuda a aprender la teoría, y es la dirigida a verificar o descubrir relaciones teóricas o conceptos. Generalmente, en estos casos aflora una perspectiva de ciencia que considera al conocimiento como algo estable y verdadero; las leyes físicas como generalizaciones inductivas; y los modelos como réplicas de la realidad. Desde esta posición, lo metodológico es sólo necesario para producir el conocimiento científico, por ello en el laboratorio se enseñan técnicas independientes de los conocimientos teóricos.

Otra forma de traducir la relación teoría-práctica en el laboratorio es considerar que la teoría se emplea en la práctica, en el sentido de que el mundo de los objetos es inseparable del mundo de los modelos. En el contexto educativo, esto implica que las actividades experimentales requieren de los estudiantes un conocimiento previo en relación a la teoría, y una toma de conciencia en cuanto al rol que ésta tendrá en los diferentes momentos del TPL (Sére, 2002). Según Kirschner (1992), el trabajo práctico se debe utilizar para enseñar y aprender la estructura sintáctica de una disciplina, más que la estructura sustantiva. Plantea tres razones o motivos válidos para ello: (a) desarrollar destrezas específicas a través de ejercicios; (b) aprender el "enfoque académico" a través de los trabajos prácticos como investigaciones, de modo que el estudiante se involucre en la resolución de problemas como lo hace un científico; y (c) tener experiencias con fenómenos.

Debe modificarse la percepción que se tiene del laboratorio de Física, dado que existen limitaciones de las actividades experimentales tradicionales, propuestas a partir de directrices fuertemente dirigidas (Dorneles, 2010). Para ello, éste debe ser considerado como un ambiente de aprendizaje. En términos generales, se puede decir que un ambiente de aprendizaje es el lugar en donde confluyen estudiantes y docentes para interactuar psicológicamente con relación a ciertos contenidos, utilizando para ello métodos y técnicas previamente establecidos con la intención de adquirir conocimientos, desarrollar habilidades, actitudes y en general, incrementar algún tipo de capacidad o competencia. González y Flores (2000), señalan que un medio ambiente de aprendizaje es el lugar donde la gente puede buscar recursos para dar sentido a las 
ideas y construir soluciones significativas para los problemas. Pensar en la instrucción como un medio ambiente destaca al 'lugar' o 'espacio' donde ocurre el aprendizaje. Los elementos de un medio ambiente de aprendizaje son: el alumno, un lugar o un espacio donde el alumno actúa, usa herramientas y artefactos para recoger e interpretar información, interactúa con otros, etcétera.

\section{LOS TRABAJOS PRÁCTICOS DE LABORATORIOS DE FÍSICA}

Los TPL's de Física, tal como se llevan a cabo en su amplia mayoría en la actualidad, plantean determinadas situaciones innecesarias que dificultan el aprendizaje. A los estudiantes se les pide frecuentemente que comprendan la naturaleza del problema y el procedimiento, que adopten la perspectiva teórica relacionada con el tema de estudio, que lean, asimilen y sigan las instrucciones del experimento, que manejen el aparato en cuestión, que recopilen los datos obtenidos, que reconozcan las diferencias entre los datos conseguidos y los resultados que "deberían haberse obtenido", que interpreten tales resultados y escriban un informe del experimento.

Estas situaciones pueden ocasionar que los estudiantes muchas veces sean incapaces de asociar la temática del TP con la teoría. Consecuentemente, es posible que adopten una de estas estrategias (Johnstone y Wham 1982): i) Adoptar un "enfoque de receta", siguiendo simplemente las instrucciones paso a paso; ii) Concentrarse en un único aspecto del experimento con la virtual exclusión del resto; iii) Mostrar un comportamiento aleatorio que les hace "estar muy ocupados sin tener nada que hacer"; iv) Mirar a su alrededor para copiar lo que están haciendo los demás; y v) Convertirse en "ayudantes" de un grupo organizado y dirigido por otros compañeros según Hodson, (1994), en una valoración crítica del Trabajo Práctico lo primero que se debería hacer es formular una serie de preguntas:

i) ¿El trabajo de laboratorio motiva a los alumnos? ¿Existen otras formas alternativas mejores de motivarlos?

ii) Los alumnos adquieren las técnicas de laboratorio a partir del trabajo práctico que realizan en la escuela? ¿La adquisición de estas técnicas es positiva desde un punto de vista educativo?

iii) ¿El trabajo de laboratorio ayuda a los alumnos a comprender mejor los conceptos científicos? iv) ¿Hay otros métodos más eficaces para conseguirlos?

v) ¿Cuál es la imagen que adquieren los alumnos sobre la ciencia y la actividad científica al trabajar en el laboratorio? ¿Se ajusta realmente esa imagen a la práctica científica habitual?

vi) ¿Hasta qué punto el trabajo práctico que efectúan los alumnos puede favorecer las denominadas "actitudes científicas"? ¿Son éstas necesarias para practicar el correcto ejercicio de la ciencia?

\section{EL ESTUDIO CINEMÁTICO INTEGRAL DE UN VOLANTE: UNA APLICACIÓN}

En este trabajo se propone el uso de las TIC's en el estudio cinemático integral de un volante. Llamamos estudio cinemático integral a aquél que permite la adquisición de datos simultáneos de la posición, velocidad y aceleración - en este caso, angulares - de un volante, donde el objetivo principal es tratar de estudiar de manera conjunta las variables cinemáticas que histórica y operacionalmente, debido a la fragmentación propia del método científico, se han analizado de manera separada y generalmente inconexa por parte de los alumnos. En esta propuesta de TPL se han empleado criterios de flexibilidad, dado que nuestro laboratorio ya disponía del volante, y al cual se le ha adaptado un mecanismo asociado al sistema de adquisición de datos en tiempo real, que ha sido adquirido a una empresa privada proveedora de equipamiento didáctico para la enseñanza de las ciencias, y tecnología y equipamiento audiovisual.

Un volante (conocido técnicamente como volante de inercia) es un disco macizo muy usado en la Mecánica, y es capaz de almacenar energía cinética, usando la inercia restante en un sistema (ver Fig. 1). La misión del volante es acumular y liberar energía transitoriamente, dado que acumula energía cinética cuando la velocidad tiende a aumentar sobre su valor de régimen y la cede cuando tiende a disminuir. Es decir, se utiliza el volante para suavizar el flujo de energía entre una fuente de potencia y su carga. El dispositivo a emplear en esta propuesta (ver Fig. 2) está conformado por el volante citado anteriormente - que pertenece al equipamiento del Laboratorio de Física de la UTN - FRSF - , el cual se conecta a un sensor de movimientos rotacionales/Encoder p/Interfase Science Workshop Pasco - Cl-6538, y éste a la Interfase USB de Altas Prestaciones para Laboratorio de Ciencias Pasco - ScienceWorkshop 750 Interface con cable de alimentación. Dicha interfase se conecta a una PC, la cual debe tener instalada el software Data Studio p/emeasure c/interfases Pasco - Cl-6870G, con licencia, el cual es suministrado con el equipamiento provisto por le empresa Pasco. 


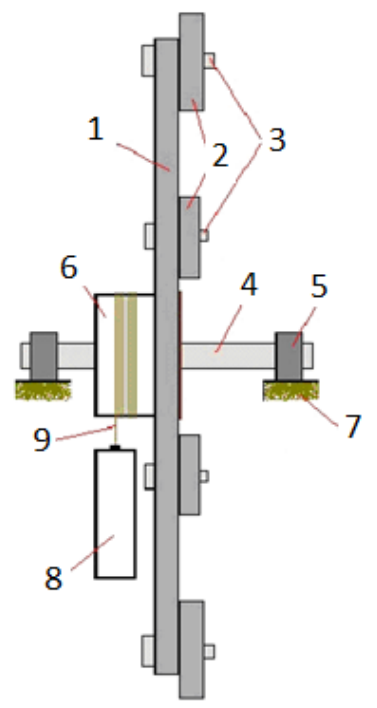

1. Disco mayor del volante.

2. Discos sobrepuestos

intercambiables para aumentar - o disminuir - el momento de inercia del volante.

3. Bulón para fijación del disco intercambiable.

4. Eje de hierro trefilado.

5. Rulemanes 6203.

6. Cilindro hueco (donde se enrolla la cuerda).

7. Soporte del equipo.

8. Pesa.

9. Cuerda transmisora de la tensión de la pesa.

Fig. 1: Volante de inercia

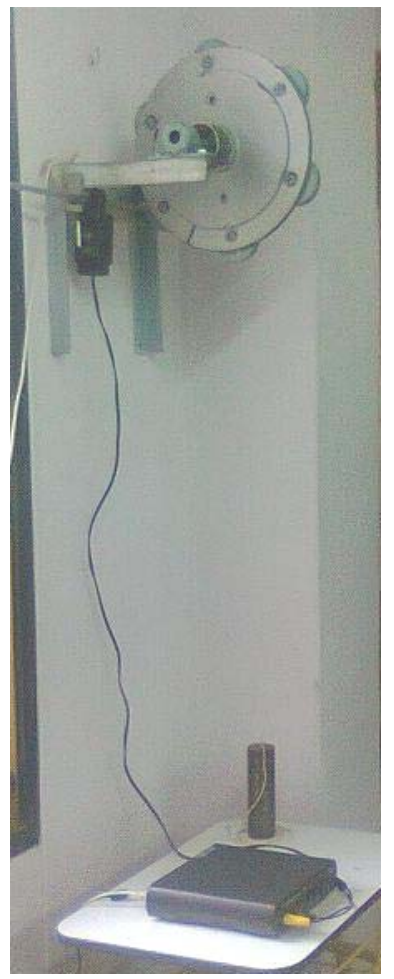

Fig. 2: Equipamiento usado en la experiencia.

La experiencia consiste en hacer uso del volante de inercia que se vincula, por un lado, con una cuerda que sostiene una pesa; y por el otro, con una cuerda inextensible y de masa despreciable a un sensor de rotación, el cual se conecta a una interfase, y ésta última a un ordenador. El software permite obtener las gráficas y/o los valores numéricos de las variables cinemáticas elegidas - en este caso, posición angular; velocidad angular; y aceleración angular - ; todas de manera simultánea y en tiempo real - . Los resultados numéricos obtenidos pueden ser transferidos a una planilla de cálculo y pueden emplearse para realizar el análisis cinemático, aunque también estas variables se pueden aprovechar para complejizar el análisis y calcular las magnitudes físicas asociadas a otras ramas de la Mecánica (por ejemplo, fuerzas y/o momentos de fuerzas - incluyendo a la cupla de rozamiento de volante - ; el momento de inercia de dicho volante; entre otras), lo que demuestra que puede hacerse un análisis muy pormenorizado asociado a la cinemática, dinámica y/o energía tanto de traslación y/o rotación. 


\section{EJECUCIÓN DEL TPL}

Operativamente, se arrolla una cuerda solidaria a la pesa en el cilindro hueco del volante, tratando de que permanezca tensa. Además, se vincula mediante una cuerda inextensible y de masa despreciable el eje del volante con el sensor de rotación. Posteriormente, se libera la pesa desde una determinada altura - igual a la longitud de la cuerda que sostiene a la pesa respecto del suelo -. Previamente, se deben medir tanto la masa de la pesa como la altura a la que se encuentra. Cuando se libera del reposo a la pesa, se acciona el dispositivo de toma de datos del programa Data Studio, a la vez que se puede iniciar el conteo del tiempo mediante un cronómetro digital, de modo de comparar los valores medidos con el programa. La experiencia se detiene cuando el volante ha cesado su movimiento. El análisis de la experiencia que se llevará a cabo en el Laboratorio de Física sobre la cinemática de rotación de un cuerpo rígido, se puede dividir en dos partes: 1. Cuando el sistema es liberado de manera que la pesa cae por acción de la gravedad produce que el volante, vinculado a la pesa a través de una cuerda arrollada en el volante, adquiera una aceleración angular; 2 . Cuando la pesa se ha desvinculado por completo del volante, es decir, la pesa ha llegado a la altura del piso, el volante queda girando con la sola perturbación de la fricción en su eje de rotación. Esto último significa que el volante en un tiempo determinado se frenará y, consecuentemente tendrá una aceleración angular negativa.

El programa puede presentar en tiempo real los valores obtenidos de posición, velocidad y aceleración angular tanto de manera gráfica como tabulados. Sugerimos el uso de ambos, para que las gráficas permitan tener una idea previa para la discusión del sistema. A continuación se presentan las gráficas halladas en nuestra experiencia (Fig. 3). Uno de los aspectos más importantes a considerar en el análisis de las magnitudes físicas es la activación de la creatividad y la propia innovación de los estudiantes.

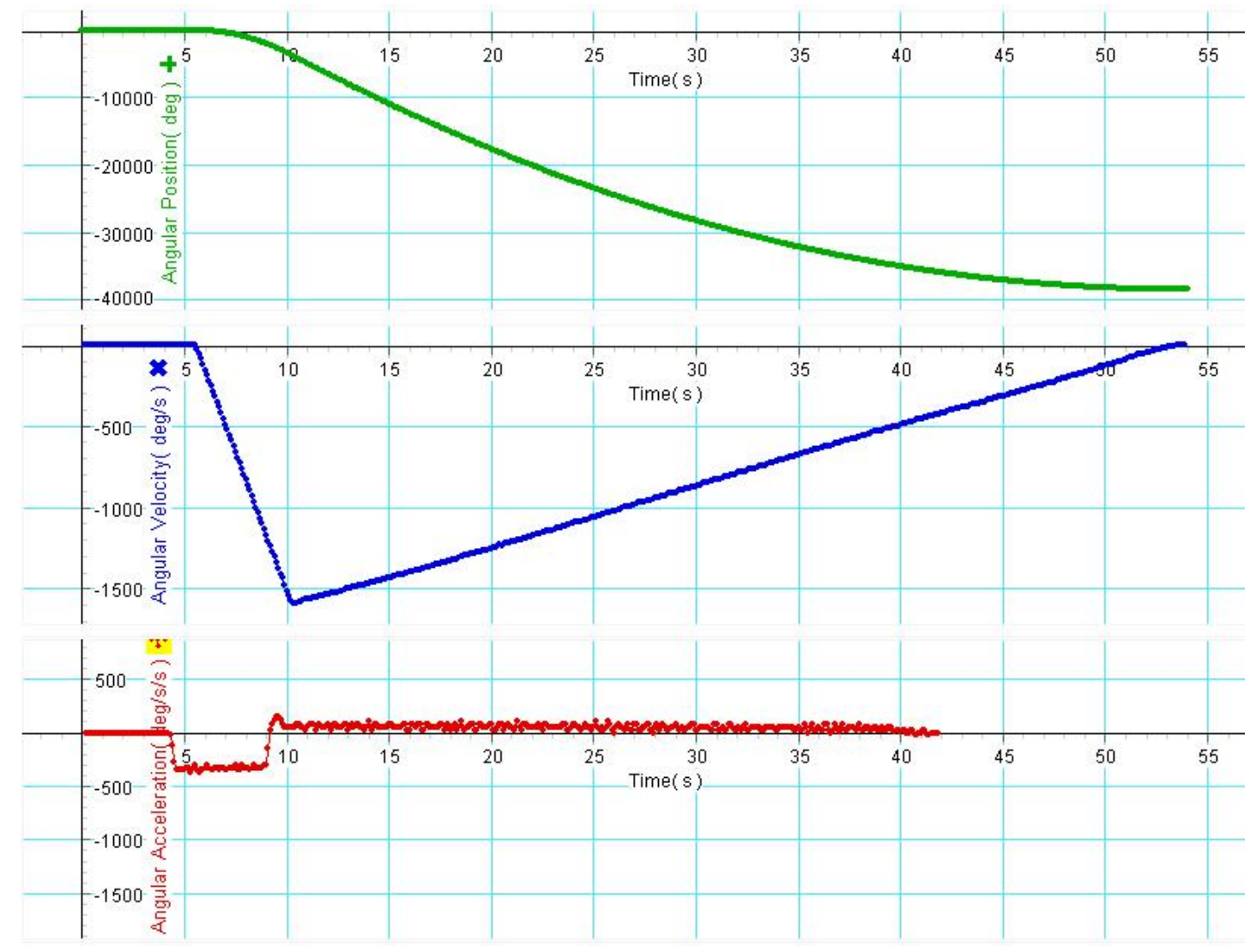

Fig. 3: Gráficas en tiempo real de posición, velocidad, y aceleración - angulares - del volante.

Para el análisis se opera sobre los valores tabulados, aprovechando que los mismos se pueden transferir a una planilla de cálculo o a un software estadístico. Sólo para mostrar una de las alternativas, se puede trabajar con la posición angular en función del tiempo y hallar la ecuación de la misma, de la cual se deben obtener los valores numéricos de las variables cinemáticas respecto a los parámetros hallados mediante estadística. A su vez, se puede verificar que por tratarse de un movimiento uniformemente acelerado, la ecuación de la posición angular respecto del tiempo debe ser una cuadrática. En la Fig. 4 se presentan en 
color azul y línea continua los datos experimentales, y en color rojo y línea quebrada la curva de regresión, junto a los parámetros de la curva de regresión - que figuran en el cuadro -. Además, se puede trabajar con la gráfica de velocidad angular respecto del tiempo, tal como la hallada en la Figura 5:

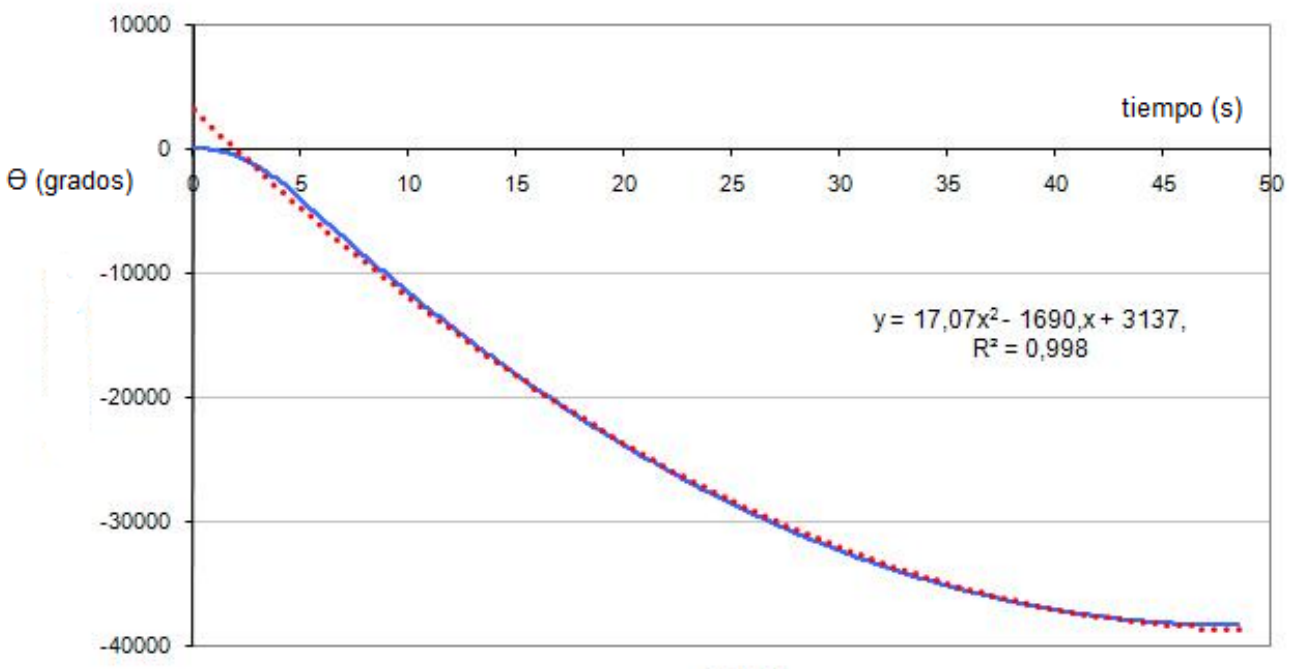

Fig. 4: Comparación entre los datos experimentales y el modelo de regresión - cuadrática -.

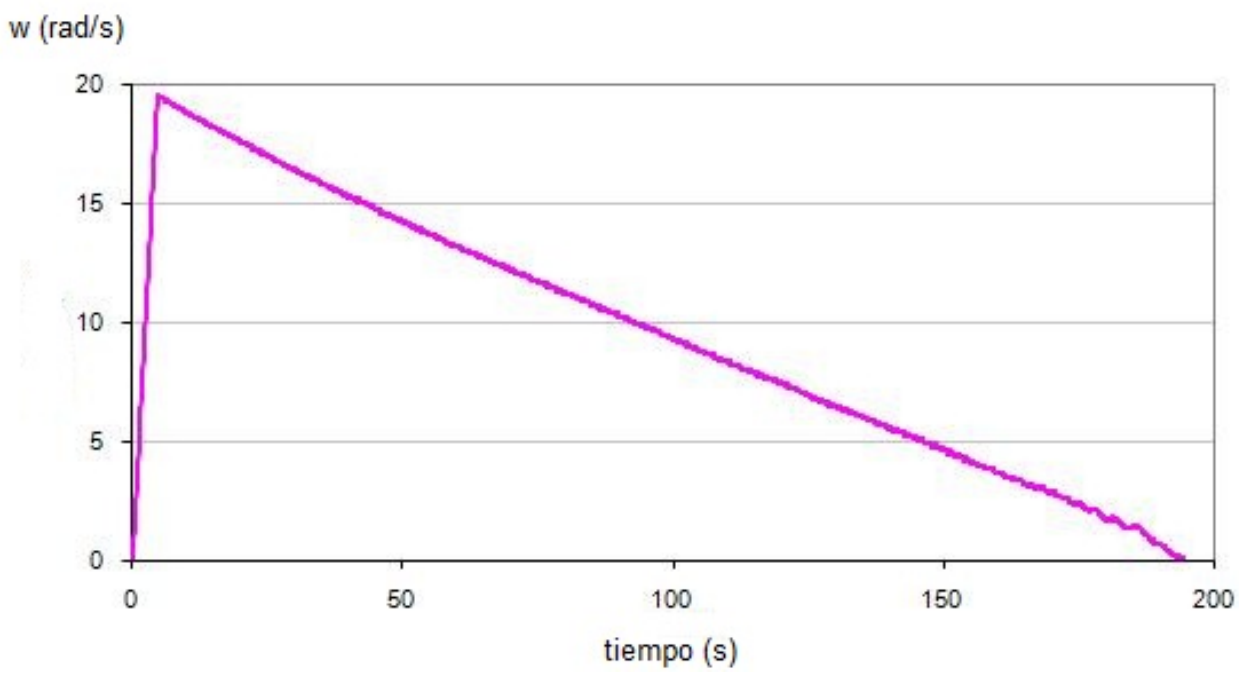

Fig. 5: Representación gráfica de la velocidad angular del volante en función del tiempo.

En la misma se puede ver cuando el sistema se libera desde el reposo (origen de la gráfica), adquiere una $\omega$ creciente hasta que se produce una discontinuidad - que es el instante cuando se libera la cuerda del volante -. Posteriormente, se desacelera angularmente hasta que cesa su movimiento. Aprovechando el uso de una planilla de cálculo, pueden hallarse las pendientes de ambas rectas, las cuales representan las aceleraciones del volante. Puede comprobarse además que se trata de un movimiento con aceleración angular constante. En estos casos se puede complementar el TPL con un análisis dinámico, estudiando los momentos de fuerza generados por la pesa, y posteriormente, por el rozamiento; o con un análisis energético.

Con el objeto de realizar un estudio pormenorizado por parte de los alumnos aprovechando las herramientas informáticas, en este TPL el - o los - docentes(s) deben diseñar preguntas tendientes a lograr un espíritu crítico y analítico en sus estudiantes, y permitir que estos últimos expongan sus resultados desarrollando una sólida argumentación. Por ejemplo: - los valores de las variables cinemáticas son negativos cuando se inicia el movimiento, mientras que en la teoría el signo fue opuesto. ¿Por qué? La respuesta es que como el giro del volante se dio en sentido horario, el programa lo toma como negativo. Simplemente, se trata de una convención. 
Otras preguntas que se pueden presentar en el cuestionario son las siguientes:

- ¿Cuáles son las variables cinemáticas obtenidas en este trabajo práctico que pueden ser empleadas para un análisis dinámico del volante? ¿Y para un análisis energético?

- ¿Cómo puede calcular el momento de inercia del volante empleado en esta experiencia?;

- ¿Cómo puede hallar la cupla de rozamiento del volante? ¿Es válido el supuesto de que se trata de una magnitud física con módulo constante? ¿Cuál es la evidencia en la realización del Trabajo Práctico?

- Realice un análisis energético del sistema.

En cualquiera de estas situaciones, los alumnos deberán realizar una investigación sobre lo que se ha medido y lo que se debe analizar para definir el estudio cinemático integral del volante. Creemos que acá se encuentra el aspecto más interesante de esta práctica, dado que los estudiantes deben tener un conocimiento muy sólido de la teoría para poder resolver distintas situaciones para arribar a resultados serios y confiables, en particular mediante el procesamiento de datos.

\section{CONCLUSIONES}

En este trabajo se ha propuesto que los TPL's en el proceso de enseñanza - aprendizaje de la Física deben ser considerados como un ambiente de aprendizaje y no como se hace muchas veces en la actualidad, donde son considerados como una actividad para "completar el cursado" o "cumplir con las condiciones para regularizar la materia", en un ambiente rutinario y con un "enfoque de receta".

Para ello, los docentes deben manejar los aspectos teóricos, pedagógicos, motivacionales y didácticos tendientes a un diseño adecuado del trabajo práctico, amén del conocimiento profundo de dicho TPL. Esta tarea se ve enormemente facilitada por el uso de materiales y equipos basados en el empleo de las TIC's, donde si bien los resultados son precisos y obtenidos de manera rápida, lo principal es "lo que queda por venir": el estudio de carácter investigativo de los resultados hallados, y bien fundamentados, con el objeto de lograr que los estudiantes tengan en cuenta la argumentación de sus conclusiones.

\section{REFERENCIAS}

Andrés, M. M., La formación del docente de física: realidad y perspectivas, Univ. Pedagógica Experimental Libertador-IPC. Caracas. Venezuela. (2002).

Ausubel, D.; Novak, J.; Hanesian, D., Psicología Educacional. Ed. Trillas, México (1983).

Barberá, O.; Valdés, P., El trabajo práctico en al enseñanza de las ciencias: una revisión. Enseñanza de las Ciencias, v. 14, n. 3, p. 365-379. (1994).

Capuano, V.; y otros cinco autores, Sobre como las "prácticas experimentales orientadas" contribuyen al cambio conceptual. Memorias V CAEDI, Mendoza, pp. 463 - 470. (2006a).

Capuano, V. y otros cinco autores, El cambio conceptual y las prácticas experimentales orientadas, en la "formación de imágenes". Memorias SIEF 8. Gualeguaychú. (2006b).

D’Ambrosio, U., Novos paradigmas de atuação e formação de docente. En: Porto, T. Redes em construção: meios de comunicação e praticas educativas. São Paulo. P. 55-77 (2003).

Dorneles, P., Integracão entre atividades computacionais e experimentais como recurso instrucional no ensino do electromagnetismo en Física Geral. Tesis de Doctorado en Ciencias, Instituto de Física, Universidad Federal do Rio Grande do Sul, Porto Alegre, Brasil (2010).

González, O., y Flores, M., El trabajo docente: enfoques innovadores para el diseño de un curso. Ed. Trillas, México. (2000).

Hodson, D., Teaching and learning about science: considerations in the philosophy and sociology of science, en Edwards, D. y Scanlon, E. (eds.), Issues in Science Education: Teaching, Learning and Assessment. (1993). 
Hodson, D., Hacia un enfoque más crítico del trabajo de laboratorio. Enseñanza de las Ciencias, v. 12, n. 3, p. 299-313. (1994).

Johnstone, A. y Wham, A., The demands of practical work, Education in Chemistry, 19, pp. 71 - 73. (1982).

Kirschner, P.A., Epistemology, practical work y academic skills in science education. Science Education, 1, 273-299. (1994).

Larkin, H.; Chabay, R., La investigación sobre la enseñanza del pensamiento científico: implicaciones para la enseñanza basada en computadoras. Recopilación de Resnick, L. y Lauren B. y Klopfer L., "Curriculum y cognición".Editorial AIQUE (1996).

Litwin. E., Las prácticas de la enseñanza y las tecnologías. Ponencia en CONTEC 2003, Universidad del museo Social Argentino - UNLP - CEDIPROE; Buenos Aires (2003).

Litwin. E., El oficio de enseñar. Ed. Paidós, Buenos Aires. (2003).

Maggio, M., El tutor en la educación a distancia. En Litwin, E. (comp.). La educación a distancia. Colección Agenda Educativa. Amorrortu Editores S.A., Buenos Aires. (2000).

Marey, M.; Amiama, C. y Alvarez, C., Metodología para la Evaluación de las Tecnologías de Información en la Docencia de Proyectos de Ingeniería. Form. Univ. [online], vol.1, n.6, pp. 3-12. ISSN 0718-5006.(2008).

Munoz-Cano, J.; Cordova, J.; y Priego, H., Dificultades y facilidades para el desarrollo et al un proceso de innovación educativa con base en las Tecnologías de la Información y Comunicación (TIC). Form. Univ. [online], vol.5, n.1, pp. 3-12. ISSN 0718-5006 (2012).

Pontes, A., Nuevas formas de aprender Física con ayuda de Internet: una experiencia educativa para aprender conceptos y procesos científicos. Revista Alambique No 29, pp. 84-94. (2001).

Richoux, H. Y Beaufils, D., La planificación de las actividades de los estudiantes en los trabajos prácticos de física: análisis de prácticas de profesores. Enseñanza de las Ciencias, 21 (1), 95-106. (2003).

San Martí, N. e Izquierdo, M., Cambio y conservación en la enseñanza de las ciencias ante las TIC. Revista Alambique $\mathrm{N}^{0}$ 29, pp. 71- 83. (2001).

Seré, M.G., Towards renewed research questions from the outcomes of the european project labwork in Science Education. Science Education, p. 625 -644. (2002).

Tobin, K.; Tippins, D.; Gallard, A. J., Research on instrucctional strategias for teaching Science. In: Gabel, D. (ed.). Handbook of Research on Science Teaching and Learning. New York: NSTA. (1994).

Ribotta, S. ; Pesetti, M. y Pereyra, S., Las Tecnologías de la Información y Comunicación (TICS) Aplicadas a la Comprensión de Gráficos en Cinemática. Form. Univ. [online], vol.2, n.5, pp. 23-30. ISSN 0718-5006. (2009). 